\title{
Condensate formation in a Bose gas
}

\author{
H.T.C. Stoof \\ Department of Physics, University of Illinois at Urbana-Champaign, 1110 West Green Street, \\ Urbana, Illinois 61801, U.S.A. and \\ Department of Theoretical Physics, Eindhoven University of Technology, P.O. Box 513, \\ 5600 MB Eindhoven, The Netherland $*$
}

\begin{abstract}
Using magnetically trapped atomic hydrogen as an example, we investigate the prospects of achieving Bose-Einstein condensation in a dilute Bose gas. We show that, if the gas is quenched sufficiently far into the critical region of the phase transition, the typical time scale for the nucleation of the condensate density is short and of $O\left(\hbar / k_{B} T_{c}\right)$. As a result we find that thermalizing elastic collisions act as a bottleneck for the formation of the condensate. In the case of doubly-polarized atomic hydrogen these occur much more frequently than the inelastic collisions leading to decay and we are lead to the conclusion that Bose-Einstein condensation can indeed be achieved within the lifetime of the gas.
\end{abstract}

PACS numbers: 67.65.+z, 32.80.Pj, 64.60.Qb

Typeset using REVTEX 


\section{INTRODUCTION}

In the last few years it has been clearly demonstrated that not only charged ions but also neutral atoms can be conveniently trapped and cooled by means of electro-magnetic fields. Although the physics of the various ingenious scenarios developed to accomplish this is already interesting in itself [1], the opportunities offered by an atomic gas sample at very low temperatures are exciting too. Examples in this respect are the performance of high-precision spectroscopy, the search for a violation of $\mathrm{CP}$ invariance by measuring the electric dipole moment of atomic cesium [2], the construction of an improved time standard based on an atomic fountain [3], and the achievement of Bose-Einstein condensation in a weakly-interacting gas.

In particular the last objective is an important motivation for studying cold atomic gases and has been pursued most vigorously with atomic hydrogen «, 4 . However, it was recently proposed that also the alkali-metal vapors cesium [6] and lithium [7] are suitable candidates for the achievement of Bose-Einstein condensation. We will nevertheless concentrate here on atomic hydrogen, because it still seems to be the most promising system for the observation of the phase transition in the near future. Moreover, it has the advantage that the atomic interaction potential is known to a high degree of accuracy. As a result we can have confidence in the fact that the scattering length is positive, which is required for the condensation to take place in the gaseous phase [8], and small enough to rigorously justify the approximations made in the following for the typical temperatures $(T \simeq 10 \mu K)$ and densities $\left(n \simeq 1 \cdot 10^{14} \mathrm{~cm}^{-3}\right)$ envisaged in the experiments.

Due to the spin of the electron and the proton, the 1s-hyperfine manifold of atomic hydrogen consists of four states which are in order of increasing energy denoted by $|a\rangle,|b\rangle$, $|c\rangle$, and $|d\rangle$, respectively. Only the $|c\rangle$ and $|d\rangle$ states can be trapped in a static magnetic

trap, because in a magnetic field they have predominantly an electron spin-up component and are therefore low-field seeking [9]. Furthermore, if we load a trap with atoms in these two hyperfine states, the $|c\rangle$ state is rapidly depopulated as a result of the much larger 
probability for collisional relaxation to the high-field seeking $|a\rangle$ and $|b\rangle$ states which are expelled from the trap. In this manner the system polarizes spontaneously and we obtain a gas of $|d\rangle$-state atoms, known as doubly spin-polarized atomic hydrogen since both the electron as well as the proton spin are directed along the magnetic field. Unfortunately, such a doubly-polarized hydrogen gas still decays due to the dipole interaction between the magnetic moments of the atoms. Although the time scale $\tau_{\text {inel }}$ for this decay is much longer than the time scale for the depopulation of the $|c\rangle$ state mentioned above, it nevertheless limits the lifetime of the gas sample to the order of seconds for the densities of interest [10].

Having filled the trap with doubly-polarized atoms, we must subsequently lower the temperature of the gas to accomplish Bose-Einstein condensation. At present it is believed that the most convenient way to achieve this is by means of conventional [11,12] or lightinduced [13] evaporative cooling. In both cases the idea is to remove, by lowering the welldepth or by photon absorption in the perimeter, the most energetic particles from the trap and thus to create momentarily a highly nonequilibrium energy distribution that will evolve into a new equilibrium distribution at a lower temperature. According to the quantum Boltzmann equation describing this process, a typical time scale for the evolution is the average time between two elastic collisions $\tau_{e l}=1 / n\langle v \sigma\rangle$, with $\langle v \sigma\rangle$ the thermal average of the relative velocity $v$ of two colliding atoms times their elastic cross section $\sigma$. Clearly, $\tau_{e l}$ must be small compared to $\tau_{\text {inel }}$ to ensure that thermal equilibrium is achieved within the lifetime of the system. As a result, the minimum temperature that can be reached by evaporative cooling is about $1 \mu K$ and indeed below the critical temperature of atomic hydrogen at a density of $1 \cdot 10^{14} \mathrm{~cm}^{-3}$.

The previous discussion appears to indicate that a typical time scale for the formation of the condensate is given by $\tau_{e l}$. However, this is not correct because simple phase-space arguments show that a kinetic equation cannot lead to a macroscopic occupation of the one-particle ground state: Considering a homogeneous system of $N$ bosons in a volume $V$, we find from the Boltzmann equation that the production rate of the condensate fraction is 


$$
\left.\frac{d}{d t} \frac{N_{\overrightarrow{0}}}{N}\right|_{i n}=C \frac{\langle v \sigma\rangle}{V}\left(1+N_{\overrightarrow{0}}\right),
$$

where $N_{\overrightarrow{0}}$ is the number of particles in the zero-momentum state and $C$ is a constant of $O(1)$. Hence, in the thermodynamic limit $(N, V \rightarrow \infty$ in such a way that their ratio $n=N / V$ remains fixed) a nonzero production rate is only possible if a condensate already exists [14 and we are forced to conclude that Bose-Einstein condensation cannot be achieved by evaporative cooling of the gas.

\section{NUCLEATION}

In the above argument we have only considered the effect of two-body collisions. It is therefore legitimate to suggest that perhaps three or more body collisions are required for the formation of the condensate, even though they are very improbable in a dilute gas [15. However, we can easily show that the same argument also applies to these processes: For a $m$ body collision that produces one particle with zero momentum we have $2 m-2$ independent momentum summations, leading to a factor of $V^{2 m-2}$. Moreover, the transition matrix element is proportional to $V \cdot V^{-m}$ due to the integration over the center-of-mass coordinate and the normalization of the initial and final state wave functions, respectively. In total the production rate for the condensate fraction is thus proportional to $V^{2 m-2}\left(V^{1-m}\right)^{2} V^{-1}\left(1+N_{\overrightarrow{0}}\right)$ or $V^{-1}\left(1+N_{\overrightarrow{0}}\right)$, which again vanishes in the thermodynamic limit if there is no nucleus of the condensed phase. As expected, the contributions from collisions that produce more than one zero-momentum particle have additional factors of $V^{-1}$ and vanish even more rapidly if $V \rightarrow \infty$

Clearly, we have arrived at a nucleation problem for the achievement of Bose-Einstein condensation which seriously endangers the success of future experiments. Fortunately, we suspect that the line of reasoning presented above is not completely rigorous because otherwise it implies that also liquid helium cannot become superfluid, in evident disagreement with our experience. Indeed, by using a kinetic equation to discuss the time evolution of the gas we have in effect neglected the buildup of coherence which is crucial for the formation of 
the condensate. Our previous argument therefore only shows that by means of evaporative cooling the gas is quenched into the critical region on a time scale $\tau_{e l}$, not that Bose-Einstein condensation is impossible. To discuss that point we need a different approach that accurately describes the time evolution of the system after the quenching by taking the buildup of coherence into account exactly. Such a nonequilibrium approach was recently developed on the basis of the Keldysh formalism and can, in the case of a dilute Bose gas, be seen as a generalization of the Landau theory of second-order phase transitions [16]. As a consequence it is useful to consider the Landau theory first. This leads to a better understanding of the more complicated nonequilibrium theory and ultimately of the physics involved in the nucleation of Bose-Einstein condensation.

\section{A. Landau theory}

As an introduction to the Landau theory of second-order phase transitions we use the example of a ferromagnetic material [17]. To be more specific we consider a cubic lattice with spins $\vec{S}_{i}$ at the sites $\{i\}$. The Hamiltonian is taken to be

$$
H=-J \sum_{\langle i, j\rangle} \vec{S}_{i} \cdot \vec{S}_{j}
$$

where $J$ is the exchange energy and the sum is only over nearest neighbors. For further convenience we also introduce the magnetization

$$
\vec{M}=\frac{1}{V} \sum_{i} \vec{S}_{i}
$$

Physically it is clear that this model has a phase transition at a critical temperature $T_{c}$ of $O\left(J / k_{B}\right)$. Above the critical temperature the thermal fluctuations randomize the direction of the spins and the system is in a disordered (paramagnetic) state having a vanishing average magnetization $\langle\vec{M}\rangle_{e q}$. However, below the critical temperature the thermal fluctuations are not large enough to overcome the directional effect of the Hamiltonian and the spins favor an

ordered (ferromagnetic) state with $\langle\vec{M}\rangle_{e q} \neq \overrightarrow{0}$. The different phases of the material are thus 
conveniently characterized by the average magnetization, which for this reason is known as the order parameter of the ferromagnetic phase transition.

In the phenomenological approach put forward by Landau the above mentioned temperature dependence of the equilibrium order parameter $\langle\vec{M}\rangle_{e q}$ is reproduced by anticipating that the free-energy density of the system at a fixed but not necessarily equilibrium value of the average magnetization has the following expansion

$$
f(\langle\vec{M}\rangle, T) \simeq f(\overrightarrow{0}, T)+\alpha(T)\langle\vec{M}\rangle^{2}+\frac{\beta(T)}{2}\langle\vec{M}\rangle^{4}
$$

for small values of $\langle\vec{M}\rangle$, and that the coefficients of this expansion behave near the critical temperature as

$$
\alpha(T) \simeq \alpha_{0}\left(\frac{T}{T_{c}}-1\right)
$$

and

$$
\beta(T) \simeq \beta_{0}
$$

respectively, with $\alpha_{0}$ and $\beta_{0}$ positive constants.

Hence, above the critical temperature $\alpha(T)$ and $\beta(T)$ are both positive. As a result the free energy is minimal for $\langle\vec{M}\rangle=\overrightarrow{0}$, which corresponds exactly to the paramagnetic phase. Moreover, for temperatures below the critical one $\alpha(T)$ is negative and the free energy is indeed minimized by a nonzero average magnetization with magnitude $\sqrt{-\alpha(T) / \beta(T)}$. Just below the critical temperature the latter equals

$$
\langle M\rangle_{e q} \simeq \sqrt{\frac{\alpha_{0}}{\beta_{0}}\left(1-\frac{T}{T_{c}}\right)},
$$

which after substitution in Eq. (四) gives rise to an equilibrium free-energy density of

$$
f\left(\langle\vec{M}\rangle_{e q}, T\right) \simeq f(\overrightarrow{0}, T)-\frac{\alpha_{0}^{2}}{2 \beta_{0}}\left(1-\frac{T}{T_{c}}\right)^{2} .
$$

Therefore, the second derivative $d^{2} f / d T^{2}$ is discontinuous at the critical temperature and the phase transition is of second order according to the Ehrenfest nomenclature. 
Note that minimizing the free energy only fixes the magnitude and not the direction of $\langle\vec{M}\rangle_{e q}$. This degeneracy is caused by the fact that the Hamiltonian in Eq. (2) is symmetric under an arbitrary rotation of all the spins $\vec{S}_{i}$. Consequently, the free energy must be symmetric under a rotation of the average magnetization and only even powers of $\langle\vec{M}\rangle$ can appear in its expansion (cf. Eq. (四). Due to this behavior the ferromagnet is a good example of a system with a spontaneously broken symmetry, i.e. although the Hamiltonian is invariant under the operations of a group, its ground state is not. In the case of a ferromagnet the symmetry group is $S O(3)$, which is broken spontaneously below the critical temperature because the average magnetization points in a certain direction. Which direction is chosen in practice, depends on the surroundings of the system and in particular on (arbitrary small) external magnetic fields that favor a specific direction.

After this summary of the Landau theory we are now in a position to introduce two time scales which turn out to be of great importance for the nucleation of Bose-Einstein condensation. To do so we consider the following experiment: Imagine that we have a piece of ferromagnetic material at some temperature $T_{1}$ above the critical temperature. Being in thermal equilibrium the material is in the paramagnetic phase with $\langle\vec{M}\rangle_{e q}=\overrightarrow{0}$. We then quickly cool the material to a new temperature $T_{2}$ below the critical temperature. If this is done sufficiently fast, the spins will have no time to react and we obtain a nonequilibrium situation in which the free energy has developed a 'double-well' structure but the average magnetization is still zero. This is depicted in Fig. [1(a). In such a situation there is a typical time scale for the relaxation of the average magnetization to its new equilibrium value $\sqrt{-\alpha\left(T_{2}\right) / \beta\left(T_{2}\right)}$, which we denote $\tau_{c o h}$.

However, in the case of magnetically trapped atomic hydrogen, the gas is isolated from its surroundings and it is not possible to perform the cooling stage mentioned above. As a result the gas has to develop the instability associated with the phase transition by itself. The time scale corresponding to this process is called $\tau_{\text {nucl }}$ and is schematically shown in

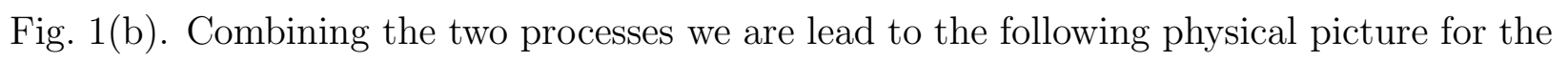
nucleation of Bose-Einstein condensation. After the quench into the critical region the gas 
develops an instability on the time scale $\tau_{\text {nucl }}$. On this time scale the actual nucleation takes place and a small nucleus of the condensate is formed, which then grows on the time scale $\tau_{c o h}$ as shown in Fig. 2. To solve the nucleation problem we are thus left with the actual determination of these two time scales. Clearly, before this can be done we need to know the correct order parameter of the phase transition.

\section{B. Order parameter}

Ever since the pioneering work of Bogoliubov [18] it is well known that the order parameter for Bose-Einstein condensation in a weakly-interacting Bose gas is a somewhat abstract quantity, which is most conveniently discussed by using the method of second quantization. In this method all many-body observables are expressed in terms of the creation and annihilation operators of a particle at position $\vec{x}$ denoted by $\psi^{\dagger}(\vec{x})$ and $\psi(\vec{x})$, respectively [19]. For

example, for a gas of particles with mass $m$ and a two-body interaction potential $V\left(\vec{x}-\vec{x}^{\prime}\right)$ the Hamiltonian equals

$$
H=\int d \vec{x} \psi^{\dagger}(\vec{x}) \frac{-\hbar^{2} \nabla^{2}}{2 m} \psi(\vec{x})+\frac{1}{2} \int d \vec{x} \int d \vec{x}^{\prime} \psi^{\dagger}(\vec{x}) \psi^{\dagger}\left(\vec{x}^{\prime}\right) V\left(\vec{x}-\vec{x}^{\prime}\right) \psi\left(\vec{x}^{\prime}\right) \psi(\vec{x})
$$

and the total number of particles is given by

$$
N=\int d \vec{x} \psi^{\dagger}(\vec{x}) \psi(\vec{x})
$$

The method is also particularly useful for a Bose system because the permutation symmetry of the many-body wave function is automatically accounted for by assuming the commutation relations $\left[\psi(\vec{x}), \psi\left(\vec{x}^{\prime}\right)\right]=\left[\psi^{\dagger}(\vec{x}), \psi^{\dagger}\left(\vec{x}^{\prime}\right)\right]=0$ and $\left[\psi(\vec{x}), \psi^{\dagger}\left(\vec{x}^{\prime}\right)\right]=\delta\left(\vec{x}-\vec{x}^{\prime}\right)$ between the creation and annihilation operators.

In the language of second quantization the order parameter for the dilute Bose gas is the expectation value $\langle\psi(\vec{x})\rangle$. Analogous to the case of the ferromagnetic phase transition, a nonzero value of this order parameter signals a spontaneously broken symmetry. Here the appropriate symmetry group is $U(1)$, since the Hamiltonian of Eq. (9) is invariant under the 
transformation $\psi(\vec{x}) \rightarrow \psi(\vec{x}) e^{i \vartheta}$ and $\psi^{\dagger}(\vec{x}) \rightarrow \psi^{\dagger}(\vec{x}) e^{-i \vartheta}$ of the field operators, whereas their expectation values are clearly not. Notice that the $U(1)$ symmetry of the Bose gas is closely related to the conservation of particle number. This is most easily seen by observing that the invariance of the Hamiltonian is due to the fact that each term in the right-hand side of Eq. (9) contains an equal number of creation and annihilation operators. The relationship can also be established in a more formal way by noting that the $U(1)$ gauge transformations are generated by the particle number operator. As we will see later on, it has important consequences for the dynamics of the order parameter.

To understand why $\langle\psi(\vec{x})\rangle$ is the order parameter associated with Bose-Einstein condensation, it is convenient to use a momentum-space description and to introduce the annihilation operator for a particle with momentum $\hbar \vec{k}$

$$
a_{\vec{k}}=\int d \vec{x} \psi(\vec{x}) \frac{e^{-i \vec{k} \cdot \vec{x}}}{\sqrt{V}}
$$

and the corresponding creation operator $a_{\vec{k}}^{\dagger}$ by Hermitian conjugation. The basis of states for the gas is then characterized by the occupation numbers $\left\{N_{\vec{k}}\right\}$. If the gas is condensed, there is a macroscopic occupation of the zero-momentum state and the relevant states are $\left|N_{\overrightarrow{0}},\left\{N_{\vec{k}}\right\}_{\vec{k} \neq \overrightarrow{0}}\right\rangle$ with only $N_{\overrightarrow{0}}$ proportional to $N$. Within this subspace of states we have

$$
\left\langle{a_{\overrightarrow{0}}}^{\dagger} a_{\overrightarrow{0}}\right\rangle=\left\langle N_{\overrightarrow{0}}\right\rangle \simeq\left\langle N_{\overrightarrow{0}}\right\rangle+1=\left\langle a_{\overrightarrow{0}} a_{\overrightarrow{0}}^{\dagger}\right\rangle
$$

and we can neglect that $a_{\overrightarrow{0}}$ and ${a_{\overrightarrow{0}}}^{\dagger}$ do not commute. As a result we can treat these operators as complex numbers [18 and say that $\left\langle{a_{\overrightarrow{0}}}^{\dagger} a_{\overrightarrow{0}}\right\rangle=\left\langle{a_{\overrightarrow{0}}}^{\dagger}\right\rangle\left\langle a_{\overrightarrow{0}}\right\rangle$ or equivalently that $\left\langle a_{\overrightarrow{0}}\right\rangle=\sqrt{N_{\overrightarrow{0}}}$. In coordinate space the latter reads $\langle\psi(\vec{x})\rangle=\sqrt{n_{\overrightarrow{0}}}$, with $n_{\overrightarrow{0}}=N_{\overrightarrow{0}} / V$ the condensate density.

The above argument essentially tells us that a sufficient condition for a nonzero value of $\langle\psi(\vec{x})\rangle$ is $\left\langle N_{\overrightarrow{0}}\right\rangle \gg 1$. Although this is intuitively appealing, it is important to point out that it is not generally true. Consider for example the ideal Bose gas [20]. In the grand canonical ensemble the total number of particles in the gas is given by

$$
N=\sum_{\vec{k}}\left\langle N_{\vec{k}}\right\rangle=\sum_{\vec{k}} \frac{1}{\zeta^{-1} e^{\beta \epsilon_{\vec{k}}}-1},
$$


where $\beta$ is $1 / k_{B} T, \epsilon_{\vec{k}}$ is the kinetic energy $\hbar^{2} \vec{k}^{2} / 2 m, \zeta$ is the fugacity $e^{\beta \mu}$ and $\mu$ is the chemical potential.

At high temperatures the fugacity is small and we are allowed to take the continuum limit of Eq. (13), which results in the equation of state

$$
n=\frac{1}{\Lambda^{3}} g_{3 / 2}(\zeta)
$$

using the thermal de Broglie wavelength $\Lambda=\sqrt{2 \pi \hbar^{2} / m k_{B} T}$ and the Bose functions $g_{n}(\zeta)$ defined by

$$
g_{n}(\zeta)=\frac{1}{\Gamma(n)} \int_{0}^{\infty} d x \frac{x^{n-1}}{\zeta^{-1} e^{x}-1}
$$

Lowering the temperature while keeping the density fixed, the fugacity increases until it ultimately reaches the value one at the critical temperature

$$
T_{0}=\frac{2 \pi \hbar^{2}}{m k_{B}}\left(\frac{n}{g_{3 / 2}(1)}\right)^{2 / 3} \simeq \frac{2 \pi \hbar^{2}}{m k_{B}}\left(\frac{n}{2.612}\right)^{2 / 3} .
$$

At this point Eq. (14) ceases to be valid because the occupation number of the zeromomentum state, which is equal to $\zeta /(1-\zeta)$, diverges and must be taken out of the discrete sum in Eq. (13) before we take the continuum limit. Moreover, we only need to treat the zero-momentum term separately because in the thermodynamic limit the chemical potential goes to zero as $V^{-1}$, whereas the kinetic energy for the smallest nonzero momentum decreases only as $V^{-2 / 3}$. Consequently, below the critical temperature the equation of state becomes

$$
n=n_{\overrightarrow{0}}+\frac{1}{\Lambda^{3}} g_{3 / 2}(1)
$$

and leads to a condensate density equal to

$$
n_{\overrightarrow{0}}=n\left(1-\left(\frac{T}{T_{0}}\right)^{3 / 2}\right) .
$$

We thus find that the average occupation number $\left\langle N_{\overrightarrow{0}}\right\rangle$ is at all temperatures given by $\zeta /(1-\zeta)$, i.e. its value in the grand canonical ensemble with the density matrix $e^{-\beta(H-\mu N)}$. 
Since this density matrix commutes with the particle number operator, we conclude that in the case of an ideal Bose gas there is a macroscopic occupation of the zero-momentum state without a spontaneous breaking of the $U(1)$ symmetry. To show more rigorously that $\langle\psi(\vec{x})\rangle_{e q}=0$ at all temperatures we determine the free-energy density of the gas as a function of the order parameter $\langle\psi(\vec{x})\rangle$. Dealing with a noninteracting system it is not difficult to obtain

$$
f(\langle\psi(\vec{x})\rangle, T)=-\mu(T)|\langle\psi(\vec{x})\rangle|^{2}
$$

for a homogeneous value of the order parameter. Because $\mu \leq 0$ the minimum is indeed always at $\langle\psi(\vec{x})\rangle=0$ and it is necessary to identify the condensate density $n_{\overrightarrow{0}}$ with the order parameter of the ideal Bose gas (cf. Eq. (18)).

Notwithstanding the previous remarks, the order parameter for Bose-Einstein condensation in a weakly-interacting Bose gas is given by $\langle\psi(\vec{x})\rangle$. This was put on a firm theoretical basis by Hugenholtz and Pines [21], who calculated the free energy as a function of the above order parameter and showed that at sufficiently low temperatures the system develops an instability that is removed by a nonzero value of $\langle\psi(\vec{x})\rangle$. In addition, they derived an exact relationship between the chemical potential and the condensate density, which turns out to be valid also in the nonequilibrium problem of interest here and is important for an understanding of how the $U(1)$ symmetry is broken dynamically.

\section{Condensation time}

We have argued that by means of evaporative cooling a doubly-polarized atomic hydrogen gas can be quenched into the critical region of the phase transition and that this kinetic part of the condensation process is described by a quantum Boltzmann equation. As a result the gas acquires on the time scale $\tau_{e l}$ an equilibrium distribution with some temperature $T$, which is slightly above the critical temperature $T_{0}$ of the ideal Bose gas because a condensate cannot be formed at this stage. 
For the study of the subsequent coherent part of the condensation process it is therefore physically reasonable to assume that at a time $t_{0}$ the density matrix $\rho\left(t_{0}\right)$ of the gas is well approximated by the density matrix of an ideal Bose gas with temperature $T$. The evolution of the order parameter $\langle\psi(\vec{x})\rangle$ for times larger than $t_{0}$ is then completely determined by the Heisenberg equation of motion

$$
i \hbar \frac{d \psi(\vec{x}, t)}{d t}=[\psi(\vec{x}, t), H]
$$

for the field operator. Substituting herein the Hamiltonian of Eq. (9) and taking the expectation value with respect to $\rho\left(t_{0}\right)$ we find

$$
i \hbar \frac{d\langle\psi(\vec{x}, t)\rangle}{d t}=\frac{-\hbar^{2} \nabla^{2}}{2 m}\langle\psi(\vec{x}, t)\rangle+\int d \vec{x}^{\prime} V\left(\vec{x}-\vec{x}^{\prime}\right)\left\langle\psi^{\dagger}\left(\vec{x}^{\prime}, t\right) \psi\left(\vec{x}^{\prime}, t\right) \psi(\vec{x}, t)\right\rangle,
$$

where the complicated part is of course the evaluation of $\left\langle\psi^{\dagger}\left(\vec{x}^{\prime}, t\right) \psi\left(\vec{x}^{\prime}, t\right) \psi(\vec{x}, t)\right\rangle$. In lowest order we simply have

$$
\begin{aligned}
\left\langle\psi^{\dagger}\left(\vec{x}^{\prime}, t\right) \psi\left(\vec{x}^{\prime}, t\right) \psi(\vec{x}, t)\right\rangle \simeq\left\langle\psi^{\dagger}\left(\vec{x}^{\prime}, t\right)\right\rangle\left\langle\psi\left(\vec{x}^{\prime}, t\right)\right\rangle\langle\psi(\vec{x}, t)\rangle & +\left\langle\psi^{\dagger}\left(\vec{x}^{\prime}, t\right) \psi\left(\vec{x}^{\prime}, t\right)\right\rangle\langle\psi(\vec{x}, t)\rangle \\
& +\left\langle\psi^{\dagger}\left(\vec{x}^{\prime}, t\right) \psi(\vec{x}, t)\right\rangle\left\langle\psi\left(\vec{x}^{\prime}, t\right)\right\rangle
\end{aligned}
$$

which after substitution into Eq. (21) leads to

$$
\begin{aligned}
\left(i \hbar \frac{d}{d t}+\frac{\hbar^{2} \nabla^{2}}{2 m}\right)\langle\psi(\vec{x}, t)\rangle= & \int d \vec{x}^{\prime} V\left(\vec{x}-\vec{x}^{\prime}\right)\left\langle\psi^{\dagger}\left(\vec{x}^{\prime}, t\right) \psi\left(\vec{x}^{\prime}, t\right)\right\rangle\langle\psi(\vec{x}, t)\rangle \\
& +\int d \vec{x}^{\prime} V\left(\vec{x}-\vec{x}^{\prime}\right)\left\langle\psi^{\dagger}\left(\vec{x}^{\prime}, t\right) \psi(\vec{x}, t)\right\rangle\left\langle\psi\left(\vec{x}^{\prime}, t\right)\right\rangle \\
& +\int d \vec{x}^{\prime} V\left(\vec{x}-\vec{x}^{\prime}\right)\left\langle\psi^{\dagger}\left(\vec{x}^{\prime}, t\right)\right\rangle\left\langle\psi\left(\vec{x}^{\prime}, t\right)\right\rangle\langle\psi(\vec{x}, t)\rangle,
\end{aligned}
$$

and thus corresponds exactly to the Hartree-Fock approximation.

To proceed we must restrict ourselves to the case of a dilute Bose gas in the quantum regime. Introducing the scattering length $a$, which is of the order of the range of the interaction, the quantum regime is characterized by $a / \Lambda \ll 1$. We therefore need to consider only $s$-wave scattering and can neglect the momentum dependence of various collisional quantities. In particular, we can replace the potential $V\left(\vec{x}-\vec{x}^{\prime}\right)$ by the contact interaction $V_{\overrightarrow{0}} \delta\left(\vec{x}-\vec{x}^{\prime}\right)$ with $V_{\overrightarrow{0}}=\int d \vec{x} V(\vec{x})$. Hence, in the Hartree-Fock approximation we obtain 


$$
\left(i \hbar \frac{d}{d t}+\frac{\hbar^{2} \nabla^{2}}{2 m}\right)\langle\psi(\vec{x}, t)\rangle=\left(2 n V_{\overrightarrow{0}}+V_{\overrightarrow{0}}|\langle\psi(\vec{x}, t)\rangle|^{2}\right)\langle\psi(\vec{x}, t)\rangle,
$$

having only the trivial solution $\langle\psi(\vec{x}, t)\rangle=0$ for a space and time-independent order parameter. Within this lowest order approximation we thus conclude that $\tau_{n u c l}=\infty$ and that the formation of a condensate will not take place.

Fortunately, it is well known that the Hartree-Fock approximation is not sufficiently accurate for a dilute Bose gas because the diluteness condition $n a^{3} \ll 1$ implies that we should consider all two-body processes, i.e. two particles must be allowed to interact also more than once. The appropriate approximation is therefore the ladder or $T$-matrix approximation and is diagrammatically explained in Fig. 3. Moreover, in the degenerate regime where the temperature $T$ is slightly larger than $T_{0}$ and the degeneracy parameter $n \Lambda^{3}$ is of $O(1)$, the condition $a / \Lambda \ll 1$ implies that also $n a \Lambda^{2} \ll 1$ or physically that the average kinetic energy of the gas is much larger than the typical interaction energy. Consequently, an accurate discussion of the nucleation of Bose-Einstein condensation in a weakly-interacting Bose gas requires an evaluation of $\left\langle\psi^{\dagger}\left(\vec{x}^{\prime}, t\right) \psi\left(\vec{x}^{\prime}, t\right) \psi(\vec{x}, t)\right\rangle$ within the $T$-matrix approximation and in zeroth order in the gas parameters $a / \Lambda$ and $n a \Lambda^{2}$.

Although it is easy to formulate this objective, to actually perform the calculation is considerably more difficult. It is most conveniently accomplished by making use of the Keldysh formalism [22] which has been reviewed by Danielewicz [23] using operator methods. For a functional formulation of this nonequilibrium theory and for the technical details of the somewhat tedious mathematics we refer to our previous papers [16]. Here we only present the final results and concentrate on the physics involved.

Due to the fact that we are allowed to neglect the (relative) momentum dependence of the $T$ matrix, the equation of motion for the order parameter $\langle\psi(\vec{x}, t)\rangle$ acquires the local form of a time-dependent Landau-Ginzburg theory

$$
\left(i \hbar \frac{d}{d t}+\frac{\hbar^{2} \nabla^{2}}{2 m}\right)\langle\psi(\vec{x}, t)\rangle=\left(S^{(+)}(t)+T^{(+)}|\langle\psi(\vec{x}, t)\rangle|^{2}\right)\langle\psi(\vec{x}, t)\rangle
$$

which is recovered from a variational principle if we use the action 
$S(\langle\psi(\vec{x}, t)\rangle, T)=\int d t \int d \vec{x}\langle\psi(\vec{x}, t)\rangle^{*}\left(i \hbar \frac{d}{d t}+\frac{\hbar^{2} \nabla^{2}}{2 m}-S^{(+)}(t)-\frac{T^{(+)}}{2}|\langle\psi(\vec{x}, t)\rangle|^{2}\right)\langle\psi(\vec{x}, t)\rangle$.

Here $S^{(+)}(t) \delta\left(t-t^{\prime}\right)$ is a good approximation for the retarded self-energy $\hbar \Sigma^{(+)}\left(\overrightarrow{0} ; t, t^{\prime}\right)$ of a hydrogen atom with zero momentum and $T^{(+)} \simeq 4 \pi \hbar^{2} a / m$ is the effective interaction between two such atoms. Clearly, the action in Eq. (26) is the desired generalization of the Landau free energy and corresponds precisely to the physical picture presented previously in Figs. 1(b) and 2(b).

Therefore, $\tau_{n u c l}$ is determined by the time dependence of the coefficient $S^{(+)}$which is shown in Fig. 团 for three different initial temperatures. If the temperature $T$ is much larger than $T_{0}, S^{(+)}(t)$ is constant and equal to $8 \pi \hbar^{2} a n / m$. In this region of the phase diagram coherent processes are negligible and the evolution of the gas is described by a Boltzmann equation. Lowering the temperature, the occupation numbers for momenta $\hbar k<O(\hbar / \Lambda)$ rise and lead to an enhancement of the coherent population of states with momenta $\hbar k<O(\hbar \sqrt{n a}) \ll O(\hbar / \Lambda)$. This is signaled by the increasing correlation length $\xi=\hbar / \sqrt{2 m S^{(+)}(\infty)}$. At the critical temperature $T_{c}=T_{0}\left(1+O\left(a / \Lambda_{0}\right)\right)$ we have $S^{(+)}(\infty)=0$ and the correlation length diverges. Below that temperature, but still above $T_{0}$ so as not to have a condensate already in the initial state, $S^{(+)}(t)$ actually changes sign and the gas develops the required instability for a Bose-Einstein condensation. The change of sign takes place at

$$
t \equiv t_{c}=t_{0}+O\left(\frac{a}{\Lambda_{c}} \frac{\hbar}{k_{B}\left(T_{c}-T\right)}\right)
$$

which shows that $\tau_{n u c l}$ is in general of $O\left(\hbar / k_{B} T_{c}\right)$ except for temperatures very close to the critical temperature. Clearly, this time scale is due to the fact that all states with momenta $\hbar k<O(\hbar / \Lambda)$ cooperate in the coherent population of the one-particle ground state.

After a small nucleus of $O\left(n\left(a / \Lambda_{c}\right)^{2}\right)$ has been formed, the subsequent buildup of the condensate density is determined by the equation of motion Eq. (25). Looking at the righthand side we immediately see that the time scale $\tau_{c o h}$ involved in this process is typically 
of $O\left(\hbar / n_{\overrightarrow{0}} T^{(+)}\right)$or equivalently of $O\left(\left(\hbar / k_{B} T_{c}\right)\left(1 / n_{\overrightarrow{0}} a \Lambda_{c}^{2}\right)\right)$. Therefore, $\tau_{c o h} \gg \tau_{n u c l}$ as anticipated in Fig. 2. The physical reason for this time scale is that after the nucleation of the phase transition the buildup of the condensate density is accompanied by a depopulation of the momentum states with $\hbar k<O\left(\hbar \sqrt{n_{\overrightarrow{0}} a}\right)$. As a result it is not difficult to show that in the limit $t \rightarrow \infty$ the condensate density is of $O\left(n a / \Lambda_{c}\right)$ and thus that $\tau_{c o h}=O\left(\left(\Lambda_{c} / a\right)^{2} \hbar / k_{B} T_{c}\right)$.

Finally, it is interesting to point out how the gas can conserve the total number of particles and apparently at the same time break the $U(1)$ gauge symmetry that is responsible for this conservation law. To that end we write the field operator $\psi(\vec{x}, t)$ as the sum of its expectation value $\langle\psi(\vec{x}, t)\rangle$ and the fluctuation $\psi^{\prime}(\vec{x}, t)$, and introduce a time-dependent chemical potential $\mu(t)$ by means of

$$
\langle\psi(\vec{x}, t)\rangle=\sqrt{n_{\overrightarrow{0}}(t)} \exp \left(-\frac{i}{\hbar} \int_{t_{0}}^{t} d t^{\prime} \mu\left(t^{\prime}\right)\right)
$$

Substituting the latter in the action of Eq. (26) and minimizing with respect to $\sqrt{n_{\overrightarrow{0}}(t)}$ gives for $t>t_{c}$

$$
n_{\overrightarrow{0}}(t)=\frac{-S^{(+)}(t)+\mu(t)}{T^{(+)}}
$$

which determines the growth of the condensate density and is in effect a nonequilibrium version of the Hugenholtz-Pines theorem [21]. Furthermore, by considering the fluctuations around $\sqrt{n_{\overrightarrow{0}}(t)}$ we can show that the chemical potential is determined by the constraint

$$
n=n_{\overrightarrow{0}}(t)+\frac{1}{V} \int d \vec{x}\left\langle\psi^{\prime \dagger}(\vec{x}, t) \psi^{\prime}(\vec{x}, t)\right\rangle
$$

enforcing the conservation of particle number at all times. In the complex plane $\langle\psi(\vec{x}, t)\rangle$ thus moves radially outward along a spiral as shown in Fig. 5. Consequently, the phase of the order parameter has never a fixed value and the $U(1)$ symmetry is not really broken dynamically. This is of course expected since the system evolves according to a symmetric Hamiltonian. 


\section{CONCLUSIONS AND DISCUSSION}

We studied the evolution of a doubly-polarized atomic hydrogen gas in a magnetic trap and showed that by means of evaporative cooling the gas can accomplish the Bose-Einstein phase transition within its lifetime $\tau_{\text {inel }}$. The condensation process proceeds under these conditions in three stages: In the first kinetic stage the gas is quenched into the critical region $T_{0}<T \leq T_{c}$. A typical time scale in this part of the evolution is given by the time between elastic collisions $\tau_{e l}$, which for a degenerate gas is of $O\left(\left(\Lambda_{c} / a\right)^{2} \hbar / k_{B} T_{c}\right)$. In the following coherent stage the actual nucleation takes place on the time scale $\tau_{\text {nucl }}=O\left(\hbar / k_{B} T_{c}\right)$ by means of a coherent population of the zero-momentum state. The small nucleus formed in this manner then grows on the much longer time scale $\tau_{c o h}=O\left(\left(\Lambda_{c} / a\right)^{2} \hbar / k_{B} T_{c}\right)$ by a depopulation of the low-momentum states, having $\hbar k<O\left(\hbar \sqrt{n_{\overrightarrow{0}}}\right)$. In the third and last stage of the evolution the Bogoliubov quasiparticles produced in the previous stage have to come into equilibrium with the condensate. This process can again be treated by a kinetic equation and was studied by Eckern [24], who found that the corresponding relaxation time $\tau_{r e l}$ is of $O\left(\left(\Lambda_{c} / a\right)^{3} \hbar / k_{B} T_{c}\right)$. In the case of atomic hydrogen this turns out to be comparable to the lifetime of the system. Summarizing, we thus have the sequence $\tau_{\text {nucl }} \ll \tau_{\text {coh }} \simeq \tau_{\text {el }} \ll \tau_{\text {rel }} \simeq \tau_{\text {inel }}$ for the various time scales involved in the phase transition. The most important requirement for the achievement of the phase transition is therefore $\tau_{e l} \ll \tau_{\text {inel }}$, which is relatively mild and should not pose an insurmountable problem for future experiments aimed at the realization of Bose-Einstein condensation.

Having arrived at this conclusion, it is necessary to discuss a recent paper by Kagan, Svistunov, and Shlyapnikov 25] that also considers the evolution of a weakly-interacting Bose gas after the removal of the most energetic atoms. In this paper the authors agree that the evolution of the gas is divided into a kinetic and a subsequent coherent stage. Moreover, their detailed study of the kinetic part of the evolution confirms our conjecture that the gas is quenched into the critical region on the time scale $\tau_{e l}$. The investigation of the coherent part, however, leads to the extreme result that a Bose-Einstein condensation cannot occur 
in a finite amount of time. To understand why this conclusion is reached we briefly present their line of thought.

At the end of the kinetic stage the gas has acquired large average occupation numbers for the states with momenta $\hbar k<\hbar k_{0}=O\left(\hbar \sqrt{n_{0} a}\right)$, where $n_{0}$ is the density of particles with these small momenta. Therefore, Kagan, Svistunov, and Shlyapnikov argue that for a study of the coherent part of the evolution we must use the initial condition

$$
\left\langle\psi\left(\vec{x}, t_{0}\right)\right\rangle=\sum_{k<k_{0}} \sqrt{\left\langle N_{\vec{k}}\right\rangle} \frac{e^{i \vec{k} \cdot \vec{x}}}{\sqrt{V}} \neq 0
$$

together with the nonlinear Schrödinger equation

$$
i \hbar \frac{d\langle\psi(\vec{x}, t)\rangle}{d t}=\left(\frac{-\hbar^{2} \nabla^{2}}{2 m}+T^{(+)}|\langle\psi(\vec{x}, t)\rangle|^{2}\right)\langle\psi(\vec{x}, t)\rangle
$$

which has the equilibrium solution $\langle\psi(\vec{x}, t)\rangle=\sqrt{n_{0}} \exp \left(-i \mu_{0} t\right)$ and $\mu_{0}=n_{0} T^{(+)}$. Consequently, all the particles that have initially momenta $\hbar k<\hbar k_{0}$ are in the limit $t \rightarrow \infty$ assumed to be in the condensate.

Linearizing the Hamiltonian of Eq. (32) around this equilibrium solution they then observe that the energy involved with a magnitude fluctuation of the order parameter is $\epsilon_{\vec{k}}+n_{0} T^{(+)}$, whereas the energy involved with a phase fluctuation is only $\epsilon_{\vec{k}}$. As a result they assert that on the time scale $\tau_{\text {ampl }}=\tau_{c o h}=O\left(\hbar / n_{0} T^{(+)}\right)$a state is formed in which the amplitude of $\langle\psi(\vec{x}, t)\rangle$ is fixed, but the phase is still strongly fluctuating because the corresponding time scale $\tau_{p h}$ is much longer and even diverges as $V^{2 / 3}$ in the thermodynamic limit. Hence, for finite times the gas is in a state with a so-called quasicondensate [26] and a real condensate is only formed in the limit $t \rightarrow \infty$.

Clearly, this physical picture of two different time scales for the amplitude and phase fluctuations of the order parameter is only applicable if these fluctuations exist independently of each other. Looking only at the Hamiltonian this indeed seems to be the case. However, a correct discussion of the fluctuations must be based on the equations of motion or equivalently the Lagrangian. The latter contains a first-order time derivative which strongly couples the amplitude and phase fluctuations. Therefore, a dilute Bose gas does 
not have two but only one dispersion relation, i.e. the well-known Bogoliubov dispersion $\sqrt{\epsilon_{\vec{k}}\left(\epsilon_{\vec{k}}+2 n_{0} T^{(+)}\right)}$, and we are lead to $\tau_{p h}=\tau_{a m p l}$. It is interesting to note that in the case of a neutral BCS-type superfluid we do have two different time scales because the Lagrangian now contains a second-order time derivative and the amplitude and phase fluctuations are indeed independent in lowest order [27,28].

An even more serious problem with the approach of Kagan, Svistunov, and Shlyapnikov is their claim that the use of the initial condition in Eq. (31) is justified because $\left\langle N_{\vec{k}}\right\rangle \gg 1$. As we have pointed out before this is not true in general. For $\langle\psi(\vec{x}, t)\rangle$ to be nonzero we must show that the system has a corresponding instability. However, within the T-matrix approximation it is not difficult to show that the instability associated with a quasicondensate is always preceded by the instability corresponding to the formation of a condensate. This implies that we always have to take Bose-Einstein condensation into account first. After that has been accomplished by means of the theory reviewed here, it is of course no longer relevant to consider the appearance of a quasicondensate.

\section{ACKNOWLEDGMENTS}

It is a great pleasure to thank Tony Leggett for various helpful discussions and for giving me the opportunity to visit the University of Illinois at Urbana-Champaign. I also benefited from conversations with Steve Girvin, Daniel Loss, Kieran Mullen, and Jook Walraven. This work was supported by the National Science Foundation through Grant No. DMR-8822688. 


\section{REFERENCES}

* Affiliation after July 31, 1993: University of Utrecht, Institute for Theoretical Physics, Princetonplein 5, 3584 CC Utrecht, The Netherlands.

[1] J. Opt. Soc. Am. B 6, No. 11 (1989), edited by S. Chu and C. Wieman.

[2] W. Bernreuther and M. Suzuki, Rev. Mod. Phys. 63, 131 (1991).

[3] A. Clairon, C. Salomon, S. Guellati, and W.D. Phillips, Europhys. Lett. 12, 683 (1990); K. Gibble and S. Chu, Phys. Rev. Lett. 70, 1771 (1993).

[4] H.F. Hess, G.P. Kochanski, J.M. Doyle, N. Masuhara, D. Kleppner, and T.J. Greytak, Phys. Rev. Lett. 59, 672 (1987); N. Masuhara, J.M. Doyle, J.C. Sandberg, D. Kleppner, T.J. Greytak, H.F. Hess, and G.P. Kochanski, Phys. Rev. Lett. 61, 935 (1988).

[5] R. van Roijen, J.J. Berkhout, S. Jaakkola, and J.T.M. Walraven, Phys. Rev. Lett. 61, 931 (1988).

[6] C. Monroe, W. Swann, H. Robinson, and C. Wieman, Phys. Rev. Lett. 65, 1571 (1990).

[7] J.J. Tollett, C.C. Bradley, and R.G. Hulet, Bull. Am. Phys. Soc. 37, 1126 (1992).

[8] H.T.C. Stoof (submitted for publication).

[9] For a review we refer to T.J. Greytak and D. Kleppner, in New Trends in Atomic Physics, edited by C. Grynberg and R. Stora (North-Holland, Amsterdam, 1984), p. 1125 and I.F. Silvera and J.T.M. Walraven, in Progress in Low Temperature Physics, edited by D.F. Brewer (North-Holland, Amsterdam, 1986), Vol. 10, p. 139.

[10] A. Lagendijk, I.F. Silvera, and B.J. Verhaar, Phys. Rev. A 33, 626 (1986); H.T.C. Stoof, J.M.V.A. Koelman, and B.J. Verhaar, Phys. Rev. B 38, 4688 (1988).

[11] J.M. Doyle, J.C. Sandberg, I.A. Yu, C.L. Cesar, D. Kleppner, and T.J. Greytak, Phys. Rev. Lett. 67, 603 (1991). 
[12] O.J. Luiten, H.G.C. Werij, I.D. Setija, T.W. Hijmans, and J.T.M. Walraven, Phys. Rev. Lett. 70, 544 (1993).

[13] I.D. Setija, H.G.C. Werij, O.J. Luiten, M.W. Reynolds, T.W. Hijmans, and J.T.M. Walraven, Phys. Rev. Lett. 70, 2257 (1993).

[14] See also E. Levich and V. Yakhot, Phys. Rev. B 15, 243 (1977) and S.G. Tikhodeev, Zh. Eksp. Teor. Fiz. 97, 681 (1990) [Sov. Phys.-JETP 70, 380 (1990)].

[15] D.W. Snoke and J.P. Wolfe, Phys. Rev. B 39, 4030 (1989).

[16] H.T.C. Stoof, Phys. Rev. Lett. 66, 3148 (1991); Phys. Rev. A 45, 8398 (1992).

[17] For a more detailed discussion see, for instance, S. Ma, Modern Theory of Critical Phenomena (Addison-Wesley, New York, 1976) and J.W. Negele and H. Orland, Quantum Many-Particle Systems (Addison-Wesley, New York, 1988).

[18] N.N. Bogoliubov, J. Phys. (Moscow) 11, 23 (1947).

[19] See, for example, A.L. Fetter and J.D. Walecka, Quantum Theory of Many-Particle Systems (McGraw-Hill, New York, 1971).

[20] K. Huang, Statistical Mechanics (Wiley, New York, 1987).

[21] N.M. Hugenholtz and D. Pines, Phys. Rev. 116, 489 (1958).

[22] L.V. Keldysh, Zh. Eksp. Teor. Fiz. 47, 1515 (1964) [Sov. Phys.-JETP 20, 235 (1965)].

[23] P. Danielewicz, Ann. Phys. (N.Y.) 152, 239 (1984).

[24] U. Eckern, J. Low Temp. Phys. 54, 333 (1984).

[25] Yu. M. Kagan, B.V. Svistunov, and G.V. Shlyapnikov, Zh. Eksp. Teor. Fiz. 101, 528 (1992) [Sov. Phys.-JETP 74, 279 (1992)]; Sov. Phys.-JETP 75, 387 (E) (1992); B.V. Svistunov, J. Moscow Phys. Soc. 1, 373 (1991).

[26] V.N. Popov, Functional Integrals in Quantum Field Theory and Statistical Physics (Rei- 
del, Dordrecht, 1983).

[27] N.N. Bogoliubov, V.V. Tolmachev, and D.N. Shirkov, New Methods in the Theory of Superconductivity (Consultants Bureau Enterprises, New York, 1959).

[28] P.W. Anderson, Phys. Rev. 112, 1900 (1958). 


\section{FIGURES}

FIG. 1. Visualization of (a) the time scale $\tau_{c o h}$ for the relaxation of the order parameter to its equilibrium value and (b) the time scale $\tau_{n u c l}$ associated with the appearance of the instability.

FIG. 2. Visualization of the time scales $\tau_{c o h}$ and $\tau_{\text {nucl }}$, using the time dependence of (a) the order parameter and (b) the coefficient $\alpha$ of the quadratic term in the free energy.

FIG. 3. Diagrammatic representation of the $T$-matrix equation. The wavy line corresponds to the interaction and the straight line to the non-interacting one-particle Green's function.

FIG. 4. Time dependence of the coefficient $S^{(+)}$for three different initial temperatures of the Bose gas.

FIG. 5. Evolution of the complex order parameter $\langle\psi(\vec{x})\rangle$, which is constrained by the requirement of particle number conservation. 